\title{
Analysis of Observer Performance in Detecting Signals with Location Uncertainty for Regularized Tomographic Image Reconstruction
}

\author{
Anastasia Yendiki and Jeffrey A. Fessler \\ Department of Electrical Engineering and Computer Science \\ University of Michigan \\ Ann Arbor, MI 48109-2122 USA \\ Email: nastazia@eecs.umich.edu
}

\begin{abstract}
Our goal is to optimize regularized image reconstruction methods for emission tomography with respect to the task of detecting small lesions in the reconstructed images. To reflect medical practice realistically, we consider the location of the lesion to be unknown. This location uncertainty significantly complicates the mathematical analysis of model observer performance. We consider model observers whose decisions are based on finding the maximum value of a local test statistic over all possible locations. Khurd and Gindi (SPIE 2004) and Qi and Huesman (SPIE 2004) described analytical approximations of the moments of the local test statistics and used Monte Carlo simulations to evaluate the localization performance of such "maximum observers". We propose here an alternative approach, where tail probability approximations developed by Adler (AAP 2000) facilitate analytical evaluation of the detection performance of these observers. We illustrate how these approximations can be used to evaluate the probability of detection (for low probability of false alarm operating points) for the Maximum Channelized Hotelling Observer. Using our analyses, one can rank and optimize image reconstruction methods without requiring time-consuming Monte Carlo simulations.
\end{abstract}

\section{INTRODUCTION}

Statistical image reconstruction methods are often used in emission tomography, as they are known to reduce noise without severely compromising resolution in the reconstructed images. These reconstruction methods typically involve one or more parameters (such as the regularization parameter in penalized-likelihood methods) that control the noise/resolution trade-off. Rather than resorting to trial-and-error, one would like to have a fast analytical method to determine reasonable choices for these parameters.

Our goal is to examine how the choice of regularization parameter in penalized-likelihood reconstruction affects the performance of observers in the task of detecting a small target signal in the reconstructed images. Human observer performance does not lend itself to analytical optimization but simple mathematical models have been developed [1, §14.3]. The detection performance of several of these model observers has been analyzed extensively for the task of detecting a signal at a known location in images produced by regularized reconstruction methods [2]-[4]. However, in clinical practice the potential location of the tumor is typically not known in advance, and so efforts have shifted to the analysis of detection tasks that involve location uncertainty [5], [6]. These efforts have focused on observers that evaluate a test statistic locally at each candidate location and compare the maximum value over all locations to a threshold. These are the observers that we will consider here as well.

In general, the exact distribution of the maximum value of correlated random fields is not known. Thus, the "bruteforce" approach to evaluating the performance of the maximum observer would be to perform time-consuming tomographic reconstructions of Monte Carlo simulated projection data and produce realizations of the maximum test statistic from the reconstructed images. To avoid this, other researchers have proposed analytical approximations of the moments of the local test statistics and used these moments to directly produce Monte Carlo simulated realizations of maximum test statistic [5], [6]. This resulted in significant time savings when compared to the brute-force method.

Here we propose an alternative approach to evaluating the performance of the maximum observer. Although the general expression for the distribution of the maximum is complicated, simple approximations of this distribution at high threshold levels do exist for correlated Gaussian random fields [7]. These approximations have been applied to the problem of detecting activation in functional neuroimaging [8]. Here we apply these approximations to tomographic reconstruction, allowing us to evaluate the probability of detection of the maximum observer at a given low probability of false alarm without performing any Monte Carlo simulations.

\section{The Detection TASK}

Let $f$ be the true object being imaged (or an approximation of the true object in $\mathbb{R}^{n_{\mathrm{p}}}$ ). This object always contains a (generally random) object background $\boldsymbol{f}_{b}$. Also, the object may or may not contain a target signal, which we assume to be highly localized in space (e.g., a lesion). When this signal is contained in the object, it is centered at one of a set of locations $\ell=1, \ldots, n_{\ell}$. We denote the target signal centered at location $\ell$ by $f_{s, \ell}$. The detection task at hand is thus to decide among 
the $n_{\ell}+1$ hypotheses $H_{0}$ and $H_{1, \ell}, \ell=1, \ldots, n_{\ell}$ :

$$
\begin{array}{rll}
H_{0} & : \quad \boldsymbol{f}=\boldsymbol{f}_{b} & \text { (signal absent) } \\
H_{1, \ell} & : \quad \boldsymbol{f}=\boldsymbol{f}_{b}+\boldsymbol{f}_{s, \ell} & \text { (signal present at location } \ell \text { ). }
\end{array}
$$

The decision is based on the observed data $\boldsymbol{y} \in \mathbb{R}^{n_{\mathrm{d}}}$.

For a tomographic imaging system and a given realization of the object $\boldsymbol{f}$, the data are characterized by mean $\mathrm{E}[\boldsymbol{y} \mid \boldsymbol{f}]=$ $\mathcal{A} \boldsymbol{f}+\boldsymbol{r}$ and covariance $\operatorname{Cov}\{\boldsymbol{y} \mid \boldsymbol{f}\}=\operatorname{diag}\{\mathcal{A} \boldsymbol{f}+\boldsymbol{r}\}$, where $\mathcal{A}$ is a linear operator modeling the system and $r$ the vector of random coincidences/scattered counts. Both $\mathcal{A}$ and $r$ are considered known. This implies that

$$
\begin{aligned}
\mathrm{E}[\boldsymbol{y}] & =\mathcal{A E}[\boldsymbol{f}]+\boldsymbol{r} \\
\operatorname{Cov}\{\boldsymbol{y}\} & =\operatorname{diag}\{\mathcal{A} \mathrm{E}[\boldsymbol{f}]+\boldsymbol{r}\}+\mathcal{A} \operatorname{Cov}\{f\} \mathcal{A}^{\prime} .
\end{aligned}
$$

The tomographic data $\boldsymbol{y}$ are noisy projections of the object $\boldsymbol{f}$, and a reconstruction step is needed to map the data from projection space back into object space. If $\mathcal{Z}$ is the operator performing this mapping, then the reconstructed image is

$$
\hat{\boldsymbol{f}}(\boldsymbol{y})=\mathcal{Z} \boldsymbol{y} .
$$

In the following, we will focus on observers that are applied on reconstructed images, since this situation better corresponds to common clinical practice.

We consider observers whose desicion rule relies on computing some scalar local test statistic $t_{\ell}=t_{\ell}(\hat{\boldsymbol{f}})$ around each of the candidate locations $\ell=1, \ldots, n_{\ell}$ in the reconstructed image. The maximum test statistic:

$$
t_{\max } \triangleq \max _{\ell=1, \ldots, n_{\ell}} t_{\ell}
$$

is then compared to a threshold $u$, which is independent of the data. If $t_{\max }>u$, it is decided that the signal is present, otherwise it is decided that the signal is absent.

Our goal is to optimize the reconstructor $\mathcal{Z}$ with respect to the performance of such an observer in the detection of the target signal. A common method of quantifying the detection performance of any observer is by tracing its Receiver Operating Characteristic (ROC) curve. For a given location $\ell$, this would be a plot of the probability of detection (deciding that the signal is present when it is actually present at $\ell$ ),

$$
P_{\mathrm{D}}(u) \triangleq \operatorname{Pr}\left(t_{\max } \geq u \mid H_{1, \ell}\right),
$$

versus the probability of false alarm (deciding that the signal is present when it is actually absent),

$$
P_{\mathrm{FA}}(u) \triangleq \operatorname{Pr}\left(t_{\max } \geq u \mid H_{0}\right) .
$$

The curve is traced by varying the decision threshold $u$.

The probabilities in (4) and (5) are difficult to obtain when the $t_{\ell}$ 's are correlated, even if their joint distribution is available, since the exact distribution of the maximum of correlated random variables has the form of a multiple integral. However, approximations of these probabilities for high values of $u$ have been developed and we will use them here to trace a portion of the ROC curve.

\section{Asymptotic Distribution of the MaXimum}

By analyzing the Euler characteristic of excursion sets, Adler has derived approximations for the distribution tails of the maximum of a correlated Gaussian random field [7]. In particular, if $t_{\max }$ is the maximum value of a $2 \mathrm{D}$ stationary Gaussian random field $T\left(x_{1}, x_{2}\right)$ with zero mean, variance $\sigma_{T}^{2}$, and autocorrelation function $R_{T}\left(x_{1}, x_{2}\right)$, then for high thresholds $u$ :

$$
\operatorname{Pr}\left(t_{\max } \geq u\right) \approx \frac{\left|\operatorname{det} \Lambda_{T}\right|^{1 / 2}|S|}{\sigma_{T}^{3}(2 \pi)^{3 / 2}} u e^{-u^{2} / 2 \sigma_{T}^{2}},
$$

where $\Lambda_{T}$ is the matrix with $i j$-th element equal to $\left\{\Lambda_{T}\right\}_{i j}=$ $-\partial^{2} R_{T}(0,0) / \partial x_{i} \partial x_{j}, i, j=1,2$, and $|S|$ is the size of the search area. According to Worsley et al. [9], this approximation has satisfactory accuracy for tail probabilities as high as 0.2.

The analysis leading to the approximation in (6) assumes a continuous random field. However, when the field is defined solely on a lattice, the results hold asymptotically as the lattice becomes finer [7]. Thus, the expression above can be used to approximate the tail distribution of the maximum test statistic in (3) when the local test statistics $t_{\ell}, \ell=1, \ldots, n_{\ell}$ can be considered stationary and their mean and autocorrelation is known.

\section{Moments of the Local Test Statistic}

We consider observers utilizing a linear channelized local test statistic [10]. Such observer models include a set of $M$ bandpass filters, attempting to mimic the human visual system, and some template, which typically has the form of a matched filter. The output of the bandpass filters is sampled at the location of interest $\ell$ to give a new feature vector $\hat{\boldsymbol{c}}_{\ell} \in \mathbb{R}^{M}$, to which the corresponding template $\boldsymbol{w}_{\ell} \in \mathbb{R}^{M}$ is then applied:

$$
\begin{aligned}
t_{\ell} & =\boldsymbol{w}_{\ell}^{\prime} \hat{\boldsymbol{c}}_{\ell}, \\
\hat{\boldsymbol{c}}_{\ell} & =\boldsymbol{C}_{\ell}^{\prime}\left(\hat{\boldsymbol{f}}-\mathrm{E}\left[\hat{\boldsymbol{f}}_{b}\right]\right)+\varepsilon_{\mathrm{int}},
\end{aligned}
$$

where $\mathcal{C}_{\ell}$ is an $1 \times M$ collection of operators. The $m$ th of these operators applies the impulse response of the $m$ th bandpass filter and samples the output at location $\ell$. The internal noise vector $\varepsilon_{\text {int }}$ models inherent uncertainty in the observer's decisions and is typically modeled as zero-mean Gaussian with covariance matrix $\boldsymbol{\Pi}_{\text {int }}$. The mean of the reconstructed background $\hat{\boldsymbol{f}}_{b} \triangleq \mathcal{Z} \mathcal{A} \boldsymbol{f}_{b}$ is subtracted from the reconstruction as in [5], [6] to signify that the observer determines the most suspect location by comparing intensities relative to the background rather than absolute intensities.

The mean of the local test statistic at location $\ell$ and the covariance at locations $\ell_{1}, \ell_{2}$ are respectively

$$
\begin{aligned}
\mathrm{E}\left[t_{\ell}\right] & =\boldsymbol{w}_{\ell}^{\prime} \mathcal{C}_{\ell}^{\prime}\left(\mathrm{E}[\hat{\boldsymbol{f}}]-\mathrm{E}\left[\hat{\boldsymbol{f}}_{b}\right]\right) \\
\operatorname{Cov}\left\{t_{\ell_{1}}, t_{\ell_{2}}\right\} & =\boldsymbol{w}_{\ell_{1}}^{\prime}\left[\mathcal{C}_{\ell_{1}}^{\prime} \mathcal{K}_{\hat{f}} \mathcal{C}_{\ell_{2}}+\delta_{\ell_{1}-\ell_{2}} \boldsymbol{\Pi}_{\text {int }}\right] \boldsymbol{w}_{\ell_{2}},
\end{aligned}
$$

where $\mathcal{K}_{\hat{f}}$ is the covariance of the reconstructed image, $\delta$ is the Kronecker delta, and we consider the internal observer noise to be uncorrelated across different locations.

Let $\overline{\boldsymbol{f}}_{b}, \mathcal{K}_{b}$ be the mean and covariance of the background respectively. In the following, we will assume that the signal 
intensity is weak with respect to the background intensity, so we can approximate $\operatorname{diag}\{\mathcal{A} \mathrm{E}[\boldsymbol{f}]+\boldsymbol{r}\} \approx \operatorname{diag}\left\{\mathcal{A} \overline{\boldsymbol{f}}_{b}+\boldsymbol{r}\right\} \triangleq \Pi$. We will also assume for simplicity that the target signal is variable in its location only and not in its shape. The reconstructors $\mathcal{Z}$ that we will consider are linear, as holds approximately with common tomographic reconstruction methods when the background is sufficiently high to render the non-negativity constraint inactive. From (1) and (2), these assumptions lead to

$$
\mathcal{K}_{\hat{f}}=\mathcal{Z}\left(\Pi+\mathcal{A} \mathcal{K}_{b} \mathcal{A}^{\prime}\right) \mathcal{Z}^{\prime}
$$

under any of the $n_{\ell}+1$ hypotheses.

Regardless of the exact distribution of the data, the local test statistics can be considered approximately Gaussian due to their linear form and the central limit theorem. Assuming that the $t_{\ell}$ 's form a correlated Gaussian random field, we will use the approximation in (6) to evaluate the probabilities of detection and false alarm in (4), (5). For a typical shift-variant tomographic system, the $t_{\ell}$ 's are not stationary. However, if we consider the system and the object background to be locally stationary over a small area around each of the locations $\ell=$ $1, \ldots, n_{\ell}$, and the target signal to be well-localized in space, we can use Fourier-space approximations for the moments of interest. Our Fourier-space approximations follow the ones that have been used in various related problems [2]-[6], [11]-[16]. Here we combine them with (6) to evaluate the probabilities in (4), (5). We illustrate the use of this approach on a specific pair of observer and reconstruction method.

\section{A. Maximum Channelized Hotelling Observer}

We focus here on the case where the local test statistic is that of the Channelized Hotelling Observer (CHO) model, whose performance has been shown to be closely correlated to that of humans in certain simple detection tasks (e.g., see [17]). The template of the $\mathrm{CHO}$ for a given location $\ell$ is

$$
\begin{aligned}
\boldsymbol{w}_{\mathrm{CHO}, \ell} & =\operatorname{Cov}\left\{\hat{\boldsymbol{c}}_{\ell}\right\}^{\dagger}\left(\mathrm{E}\left[\hat{\boldsymbol{c}}_{\ell} \mid H_{1, \ell}\right]-\mathrm{E}\left[\hat{\boldsymbol{c}}_{\ell} \mid H_{0}\right]\right) \\
& =\left[\mathcal{C}_{\ell}^{\prime} \mathcal{K}_{\hat{f}} \mathcal{C}_{\ell}+\boldsymbol{\Pi}_{\mathrm{int}}\right]^{\dagger} \mathcal{C}_{\ell}^{\prime} \mathcal{Z} \mathcal{A} \boldsymbol{f}_{s, \ell},
\end{aligned}
$$

with $\mathcal{K}_{\hat{f}}$ as defined in (9) and " $\dagger$ " denoting a pseudo-inverse. Thus, our observer here is the Maximum CHO (MaCHO), which applies the above template at each candidate location within a search area of interest and then compares the maximum value to a threshold.

\section{B. Penalized Weighted Least Squares Reconstructor}

We would like to evaluate the performance of the $\mathrm{MaCHO}$ when it is applied to images produced by regularized reconstruction methods. For simplicity we focus here on unconstrained Penalized Weighted Least Squares (PWLS) reconstruction. It is straightforward to extend such analysis to general penalized likelihood [18]. Unconstrained PWLS with the usual $\Pi^{-1}$ weighting reduces to

$$
\begin{aligned}
\hat{\boldsymbol{f}}(\boldsymbol{y}) & =\underset{\boldsymbol{f}}{\arg \min }\left\{\frac{1}{2}\|\boldsymbol{y}-\mathcal{A} \boldsymbol{f}\|_{\boldsymbol{\Pi}^{-1 / 2}}^{2}+\frac{1}{2} \beta \boldsymbol{f}^{\prime} \mathcal{R} \boldsymbol{f}\right\} \\
& =(\mathcal{F}+\mathcal{R})^{-1} \mathcal{A}^{\prime} \boldsymbol{\Pi}^{-1} \boldsymbol{y}=\boldsymbol{Z} \boldsymbol{y}
\end{aligned}
$$

where $\mathcal{F} \triangleq \mathcal{A}^{\prime} \Pi^{-1} \mathcal{A}$ is a Fisher information operator and $\mathcal{R}$ is a roughness penalty operator.

\section{Moments of the Local Test Statistic for MaCHO/PWLS}

Substituting (9), (10) and (11) in (7) and (8) yields:

$$
\begin{aligned}
\mathrm{E}\left[t_{\ell} \mid H_{0}\right]= & 0 \\
\mathrm{E}\left[t_{\ell_{1}} \mid H_{1, \ell_{2}}\right]= & \boldsymbol{f}_{s, \ell_{1}}^{\prime} \mathcal{F}(\mathcal{F}+\mathcal{R})^{-1} \mathcal{C}_{\ell_{1}} \\
\cdot & {\left[\mathcal{C}_{\ell_{1}}^{\prime} \mathcal{K}_{\hat{f}} \mathcal{C}_{\ell_{1}}+\boldsymbol{\Pi}_{\mathrm{int}}\right]^{\dagger} } \\
\cdot & \mathcal{C}_{\ell_{1}}^{\prime}(\mathcal{F}+\mathcal{R})^{-1} \mathcal{F} \boldsymbol{f}_{s, \ell_{2}} \\
\operatorname{Cov}\left\{t_{\ell_{1}}, t_{\ell_{2}}\right\}= & \boldsymbol{f}_{s, \ell_{1}}^{\prime} \mathcal{F}(\mathcal{F}+\mathcal{R})^{-1} \mathcal{C}_{\ell_{1}} \\
\cdot & {\left[\mathcal{C}_{\ell_{1}}^{\prime} \mathcal{K}_{\hat{f}} \mathcal{C}_{\ell_{1}}+\boldsymbol{\Pi}_{\text {int }}\right]^{\dagger} } \\
\cdot & {\left[\mathcal{C}_{\ell_{1}}^{\prime} \mathcal{K}_{\hat{f}} \mathcal{C}_{\ell_{2}}+\delta_{\ell_{1}-\ell_{2}} \boldsymbol{\Pi}_{\mathrm{int}}\right] } \\
\cdot & {\left[\mathcal{C}_{\ell_{2}}^{\prime} \mathcal{K}_{\hat{f}} \mathcal{C}_{\ell_{2}}+\boldsymbol{\Pi}_{\mathrm{int}}\right]^{\dagger} } \\
& \mathcal{C}_{\ell_{2}}^{\prime}(\mathcal{F}+\mathcal{R})^{-1} \mathcal{F} \boldsymbol{f}_{s, \ell_{2}},
\end{aligned}
$$

where $\mathcal{K}_{\hat{f}}=(\mathcal{F}+\mathcal{R})^{-1}\left(\mathcal{F}+\mathcal{F} \mathcal{K}_{b} \mathcal{F}\right)(\mathcal{F}+\mathcal{R})^{-1}$ for PWLS reconstruction.

To get computationally tractable expressions for the moments above, we will use local shift-invariance approximations [11] to write

$$
\begin{aligned}
\mathrm{E}\left[t_{\ell_{1}} \mid H_{1, \ell_{2}}\right] & \approx \boldsymbol{X}^{\prime} \boldsymbol{G} \boldsymbol{T}\left[\boldsymbol{T}^{\prime} \boldsymbol{H} \boldsymbol{T}+n_{\mathrm{p}} \boldsymbol{\Pi}_{\mathrm{int}}\right]^{\dagger} \boldsymbol{T}^{\prime} \boldsymbol{G} \boldsymbol{X}(12) \\
\operatorname{Cov}\left\{t_{\ell_{1}}, t_{\ell_{2}}\right\} & \approx \boldsymbol{X}^{\prime} \boldsymbol{G} \boldsymbol{T}\left[\boldsymbol{T}^{\prime} \boldsymbol{H} \boldsymbol{T}+n_{\mathrm{p}} \boldsymbol{\Pi}_{\mathrm{int}}\right]^{\dagger} \\
& \cdot\left[\boldsymbol{T}^{\prime} \boldsymbol{H} \boldsymbol{E}+n_{\mathrm{p}} \boldsymbol{\Pi}_{\mathrm{int}}\right] \\
& \cdot\left[\boldsymbol{T}^{\prime} \boldsymbol{H} \boldsymbol{T}+n_{\mathrm{p}} \boldsymbol{\Pi}_{\mathrm{int}}\right]^{\dagger} \boldsymbol{T}^{\prime} \boldsymbol{G} \boldsymbol{X}
\end{aligned}
$$

where $\boldsymbol{E}$ is a diagonal matrix containing the complex exponential that corresponds to the shift between locations $\ell_{2}, \ell_{1}$,

$$
\begin{aligned}
& \boldsymbol{G} \triangleq \operatorname{diag}\left\{\frac{\lambda_{k}}{\lambda_{k}+\omega_{k}}\right\}, \\
& \boldsymbol{H} \triangleq \operatorname{diag}\left\{\frac{\lambda_{k}\left(1+\lambda_{k} \mu_{k}\right)}{\left(\lambda_{k}+\omega_{k}\right)^{2}}\right\},
\end{aligned}
$$

and $\left\{\lambda_{k}\right\},\left\{\omega_{k}\right\},\left\{\mu_{k}\right\}$ are the frequency responses of $\mathcal{F}, \mathcal{R}, \mathcal{K}_{b}$ respectively (locally around the location of interest). Finally, $\boldsymbol{X}$ is the frequency response of the target signal $\boldsymbol{f}_{s, \ell}$ and the columns of $\boldsymbol{T}$ contain the frequency responses of the channels $C_{\ell}$ (calculated as if the signal and channels were centered at the $(0,0)$ point of the Fourier transform).

The expressions above require only the inversion of an $M \times M$ matrix, which is typically a significant reduction in dimensionality. In the special case where the channels of the MaCHO have non-overlapping passpands, the columns of $\boldsymbol{T}$ are orthogonal and the expressions above are particularly fast to compute, with no matrix inversion required.

\section{MaChO Detection Performance}

We use the approximation in (6) to calculate the probabilities of detection (4) and false alarm (5) of the $\mathrm{MaCHO}$ at high detection thresholds $u$. We write respectively:

$$
\begin{aligned}
P_{\mathrm{D}}(u) & \approx \frac{\left|\operatorname{det} \Lambda_{T}\right|^{1 / 2}|S|}{\sigma_{T}^{3}(2 \pi)^{3 / 2}}\left(u-\mu_{T}\right) e^{-\left(u-\mu_{T}\right)^{2} / 2 \sigma_{T}^{2}}(14) \\
P_{\mathrm{FA}}(u) & \approx \frac{\left|\operatorname{det} \Lambda_{T}\right|^{1 / 2}|S|}{\sigma_{T}^{3}(2 \pi)^{3 / 2}} u e^{-u^{2} / 2 \sigma_{T}^{2}}
\end{aligned}
$$




\section{Phantom and search area}

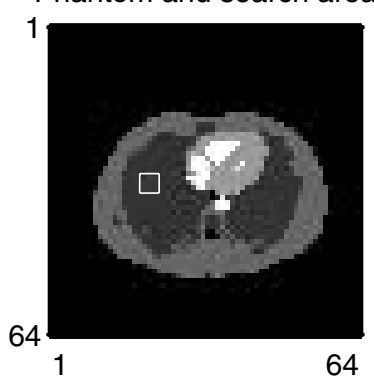

Profile of phantom and lesion

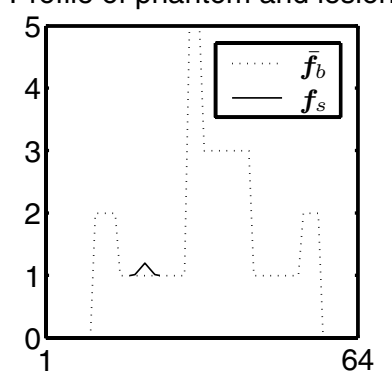

Fig. 1. Mean background and search area (left) and profile through the mean background with the target signal superimposed (right).

where we obtain $\sigma_{T}$ and $\Lambda_{T}$ from (13), and $\mu_{T}$ from (12). In particular, we use $\mu_{T} \approx \mathrm{E}\left[t_{\ell} \mid H_{1, \ell}\right]$, where $\ell$ the center of the search area, although $\mu_{T}$ in fact is not constant throughout the search area. This assumes that the maximum most likely occurs in the search area center, and thus is an approximation that needs to be validated.

\section{PREliminary Results}

We provide an example of using the above approximations to choose the regularization parameter $\beta$ for PWLS reconstruction. We consider the case where $\mathcal{A}$ corresponds to a 2 -D SPECT system model with depth-dependent system blur and the target signal $f_{s, \ell}$ has a known Gaussian shape but unknown location $\ell$. We assume that the background $f_{b}$ has a Gaussian autocorrelation function (ACF) and the mean background $\bar{f}_{b}$ is the anthropomorphic phantom shown in figure 1. Also shown in figure 1 is the square search area considered by the observer. Any pixel in this area is a candidate location for the target signal. The $\mathrm{CHO}$ in this example has 4 channels with ideal non-overlapping radially symmetric passbands and, for now, no internal noise. We consider PWLS with first-order quadratic regularization and various values of the regularization parameter $\beta$.

We use our analytical approximations (14) and (15) to calculate $P_{\mathrm{D}}(u)$ and $P_{\mathrm{FA}}(u)$ for different values of the threshold $u$. We repeat this for each value of $\beta$. In figure 2 we plot $P_{\mathrm{D}}$ vs. $\beta$ for a fixed $P_{\mathrm{FA}}=0.02$ and we show reconstructions of a noisy Poisson data set with $\log _{2} \beta=0$ (which achieves peak $\left.P_{\mathrm{D}}\right)$ and with a lower and a higher $\beta$.

Next, we use our analytical approximations to trace the highthreshold segment of the ROC curve for different values of $\beta$. The left plot in figure 3 shows the resulting ROC curves for $\log _{2} \beta=0$ as well as for two extreme values of $\beta$. To evaluate the accuracy of our approximations, we also obtain empirical ROC curves by generating samples from the exact Gaussian distribution of the $t_{\ell}$ 's. The right plot in figure 3 compares the analytical and empirical curves for $\log _{2} \beta=0$. Finally, we provide separate plots of the analytical and empirical $P_{\mathrm{FA}}(u)$ and $P_{\mathrm{D}}(u)$ vs. the threshold $u$ for $\log _{2} \beta=0$ in figure 4 .

Plots like the one in figure 2 can be used to choose the regularization parameter $\beta$ for each pixel in the image so as to
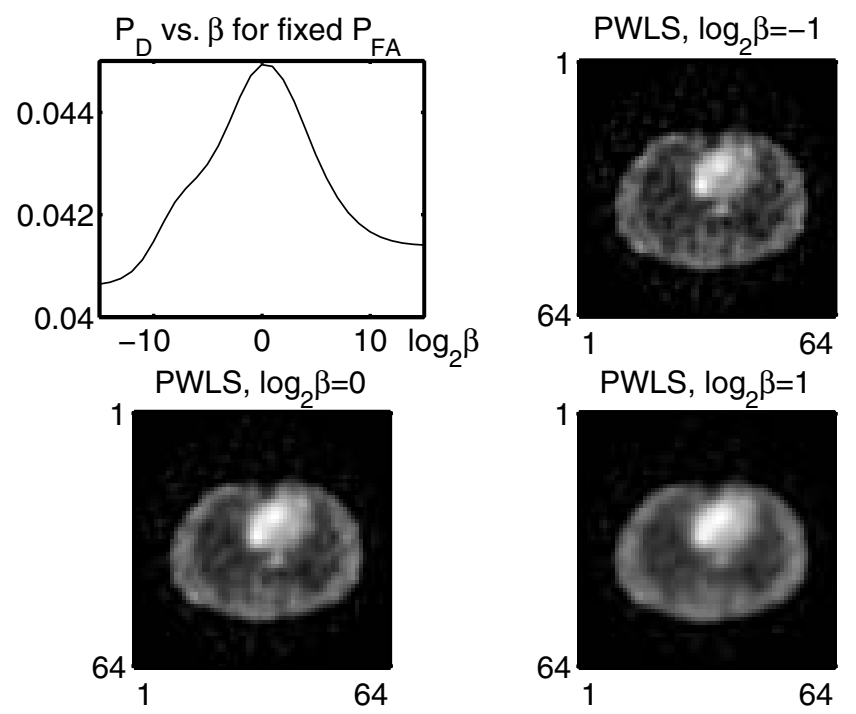

Fig. 2. Plot of $P_{\mathrm{D}}$ vs. $\beta$ for a fixed $P_{\mathrm{FA}}=0.02$ and PWLS reconstructions of a noisy Poisson data set with $\log _{2} \beta=0$ (which achieves peak $P_{\mathrm{D}}$ ) and with a lower and a higher $\beta$.
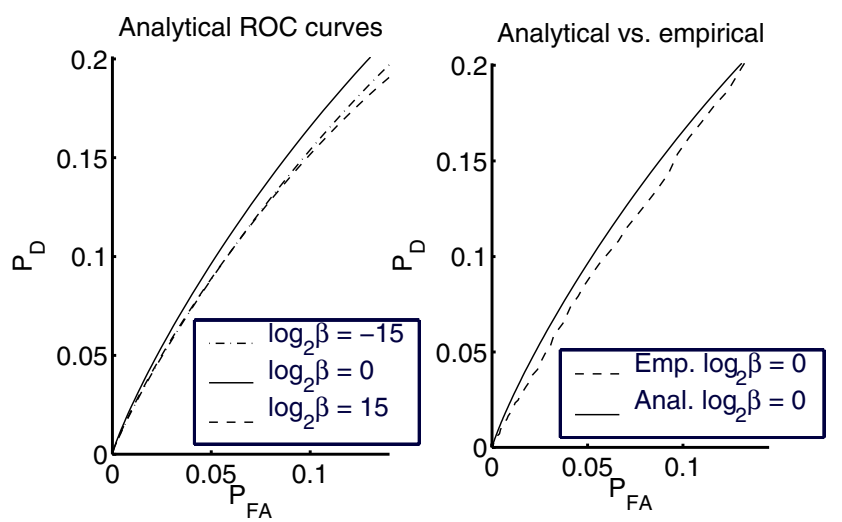

Fig. 3. High-threshold segment of the ROC, as obtained from analytical approximations with three different values of $\beta$ (left) and as obtained both from analytical approximations and empirical simulations for $\log _{2} \beta=0$ (right).

optimize the probability of detection for a given low probability of false alarm in a small region around that pixel. By increasing the probability of detection at some probability of false alarm, we increase the area under the entire ROC curve. This is significantly faster than performing Monte Carlo simulations to directly optimize the area under the ROC or LROC curve.

\section{FUTURE WORK}

To refine the approximations in (14) and (15), we intend to examine the effect of additional terms of lower order in the approximations that have been derived by Adler.

In the example of the previous section, we have not included internal noise in the observer model. The reason is that internal noise manifests itself as an impulse at the point $(0,0)$ of the autocorrelation function $R_{T}$ and thus issues arise with taking 

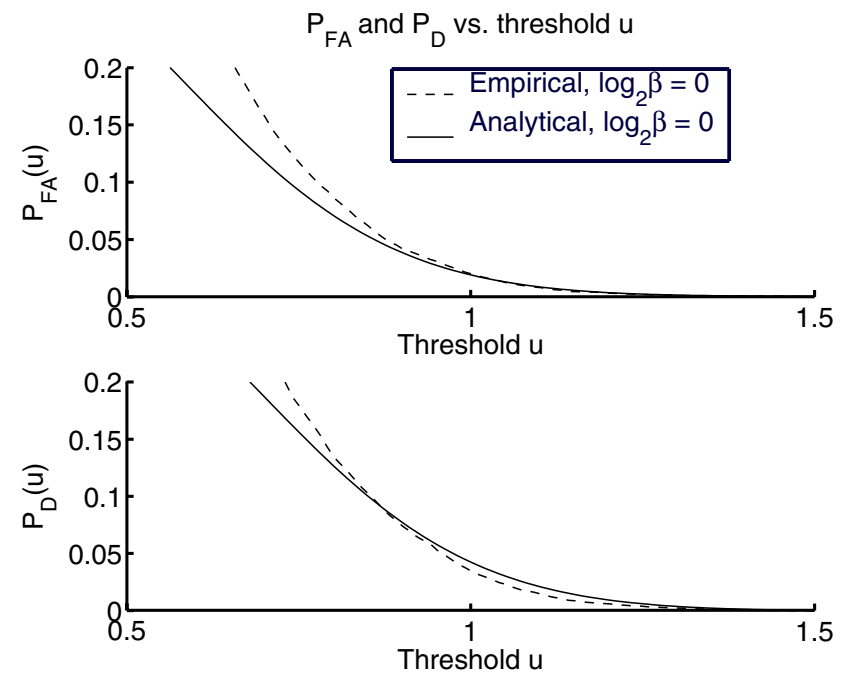

Fig. 4. Empirical and analytical probabilities of false alarm and detection vs. the detection threshold.

its derivative. We intend to investigate further the inclusion of internal noise in our approximations.

As mentioned previously, in analyzing the probability of detection we have crudely approximated the mean signal as constant throughout the search area. This approximation is acceptable when the maximum value of the test statistic under the signal-present hypothesis is most often observed at the true location of the signal peak. We are currently investigating alternative approximations of the probability of detection that may weaken this assumption.

Finally, as empirical simulations can show, there is increased probability that the maximum of the correlated $t_{\ell}$ 's occurs at the search area corners. This gives rise to the question whether the current form of the maximum observer is a good representation of a human observer or whether it needs to be adjusted.

\section{ACKNOWLEDGMENT}

This work is being supported in part by NIH grant EB-00218 and by NSF grant BES-9982349.

\section{REFERENCES}

[1] H. H. Barrett and K. J. Myers, Foundations of image science. New York: Wiley, 2003.

[2] Y. Xing, I.-T. Hsiao, and G. Gindi, "Rapid calculation of detectability in Bayesian single photon emission computed tomography," Phys. Med. Biol., vol. 48, no. 22, pp. 3755-74, Nov. 2003.

[3] A. Yendiki and J. A. Fessler, "Analytical approach to channelized Hotelling observer performance for regularized tomographic image reconstruction," in Proc. IEEE Intl. Symp. Biomedical Imaging, 2004, pp. $360-3$.

[4] J. Qi, "Analysis of lesion detectability in Bayesian emission reconstruction with nonstationary object variability," IEEE Tr. Med. Im., vol. 23 , no. 3, pp. 321-9, Mar. 2004.

[5] P. K. Khurd and G. R. Gindi, "LROC model observers for emission tomographic reconstruction," in Proc. SPIE 5372, Medical Imaging 2004. Image Perception, Observer Performance, and Technology Assessment, 2004, pp. 509-20.

[6] J. Qi and R. H. Huesman, "Fast approach to evaluate MAP reconstruction for lesion detection and localization," in Proc. SPIE 5372, Medical Imaging 2004: Image Perception, Observer Performance, and Technology Assessment, 2004, pp. 273-82.

[7] R. J. Adler, "On excursion sets, tube formulas and maxima of random fields," Ann. Appl. Probab., vol. 10, no. 1, pp. 1-74, 2000.

[8] K. J. Worsley, "Detecting activation in fMRI data," Stat. Meth. Med. Res., vol. 12, no. 5, pp. 401-18, Oct. 2003.

[9] K. J. Worsley, S. Marrett, P. Neelin, A. C. Vandal, K. J. Friston, and A. C. Evans, "A unified statistical approach for determining significan signals in images of cerebral activation," Hum. Brain Map., vol. 4, no. 1, pp. 58-73, 1996.

[10] K. J. Myers and H. H. Barrett, "Addition of a channel mechanism to the ideal-observer model," J. Opt. Soc. Am. A, vol. 4, no. 12, pp. 2447-57, Dec. 1987.

[11] J. A. Fessler and W. L. Rogers, "Spatial resolution properties of penalizedlikelihood image reconstruction methods: Space-invariant tomographs," IEEE Tr. Im. Proc., vol. 5, no. 9, pp. 1346-58, Sept. 1996.

[12] J. A. Fessler and S. D. Booth, "Conjugate-gradient preconditioning methods for shift-variant PET image reconstruction," IEEE Tr. Im. Proc., vol. 8, no. 5, pp. 688-99, May 1999.

[13] J. Qi and R. M. Leahy, "A theoretical study of the contrast recovery and variance of MAP reconstructions with applications to the selection of smoothing parameters," IEEE Tr. Med. Im., vol. 18, no. 4, pp. 293-305, Apr. 1999.

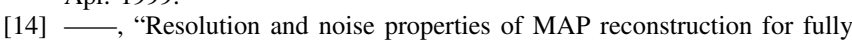
3D PET," IEEE Tr. Med. Im., vol. 19, no. 5, pp. 493-506, May 2000.

[15] J. W. Stayman and J. A. Fessler, "Regularization for uniform spatial resolution properties in penalized-likelihood image reconstruction," IEEE Tr. Med. Im., vol. 19, no. 6, pp. 601-15, June 2000.

[16] J. A. Fessler, "Analytical approach to regularization design for isotropic spatial resolution," in Proc. IEEE Nuc. Sci. Symp. Med. Im. Conf., 2003, to appear.

[17] C. K. Abbey and H. H. Barrett, "Human- and model-observer performance in ramp-spectrum noise: effects of regularization and object variability," J. Opt. Soc. Am. A, vol. 18, no. 3, pp. 473-88, Mar. 2001.

[18] J. A. Fessler, "Mean and variance of implicitly defined biased estimators (such as penalized maximum likelihood): Applications to tomography," IEEE Tr. Im. Proc., vol. 5, no. 3, pp. 493-506, Mar. 1996. 